\title{
Reviewer's comment concerning "Posterior transdural discectomy: a new approach for the removal of a central thoracic disc herniation." (doi:10.1007/s00586-011-1990-4 by H.M. Coppes et al.)
}

\author{
S. M. H. Mehdian
}

Accepted: 16 August 2011/Published online: 9 September 2011

(C) Springer-Verlag 2011

Having carefully reviewed the paper, as a reviewer and senior spine surgeon with over 25 years of experience, I have serious concerns about the perilous nature of the operative technique advocated in this article. I believe that this technique should not be recommended for a thoracic disc herniation as it violates the basic principles of spinal surgery.

The paper has several drawbacks, which I have highlighted. Having performed somewhere in the region of 20 thoracic disc excisions, I believe the safest approach with the lowest risk of neurological sequelae is via a thoracotomy. The paper lacks a large series to support the safe use of this technique by most spinal surgeons who have limited experience with intra-dural work, especially in the thoracic region. Also, I firmly believe that intra-dural exposure of the thoracic spinal cord is fraught with a high risk of neurological injury with significant implications to the patient on a long term and hence should not be done. There are several other issues with this technique such as the use of drill adjacent to the exposed cord (risking both mechanical and thermal damage) and the use of fibrin glue to seal the anterior dural opening (given that the product is not licensed to be used for this purpose due to its swelling properties thereby risking cord compression).

The European Spine Journal has several million readers and I have grave concerns about the implications of publishing this article in a highly indexed 'peer reviewed' journal. As the peer group reviewing article for publication, I believe we have a responsibility to the spine fraternity to accept publications which advocate safe proven techniques, which does not compromise patient safety in any manner.
S. M. H. Mehdian ( $\square)$

The Centre for Spinal Studies and Surgery, Nottingham

University Hospital, Queen's Medical Centre Campus,

Nottingham NG7 2UH, UK

e-mail: hossein.mehdian@nuh.nhs.uk 